\title{
Flavonoid Components of Different Color Magnolia Flowers and Their Relationship to Cultivar Selections
}

\author{
Ninghang Wang ${ }^{1}$, Chao Zhang, Sainan Bian, Pengjie Chang, \\ Lingjuan Xuan, Lijie Fan, Qin Yu, Zhigao Liu, and Cuihua Gu \\ College of Landscape and Architecture, Zhejiang Agriculture and Forestry \\ University, Hangzhou 311300, China
}

Shouzhou Zhang
Shenzhen Fairy Lake Botanical Garden, Shenzhen 518004, China

Yaling Wang

Xian Botanical Garden of Shaanxi Academy of Science, Xian 710061, China

Yamei Shen ${ }^{1,2}$

College of Landscape and Architecture, Zhejiang Agriculture and Forestry University, Hangzhou 311300, China

Additional index words. Magnolia, HPLC-DAD/ESI-MS, Cyanidin, Peonidin, flavonols

Abstract. Magnolia (Magnoliaceae) is widely cultivated for its beauty; however, despite this, the components of the different flower colors in Magnolia have not been elucidated. In this study, the color parameters of 10 Magnolia petals with different colors were measured by the Royal Horticultural Society Color Chart (RHSCC) and a color reader CR-10. The composition and content of the flavonoids in the petals were analyzed by high-performance liquid chromatography coupled with diode array detection (HPLC-DAD) as well as HPLC with electrospray ionization and mass spectrometry (HPLC-ESI-MS ${ }^{2}$ ). All results showed that the 10 petals were divided into four color groups. Regarding the flavonoid composition, four types of anthocyanins, including Cyanidin-glucosyl-rhamnoside (Cy-GR), Cyanidinglucosyl-rhamnosyl-glucoside (Cy-GRG), Peonidin-glucosyl-rhamnoside (Pn-GR), and Peonidin-glucosyl-rhamnosyl-glucoside (Pn-GRG), were identified, as well as 10 types of flavonols. The flavonols included isorhamnetin, quercetin, kaempferol, and their glycosides, which included rutinoside, rhamnose, and glucoside. Cyanidin and peonidin make Magnolia petals appear red-purple and purple, respectively, and the flavonols perform as evident auxiliary pigments, particularly quercetin. The Magnolia cultivar flower phenotypes sampled in this study differed by changes in their existing flavonoid content rather than by the appearance of new flavonoids. Consequently, this study provides a reference for further revealing the basis of Magnolia flower color and provides clues for color breeding.

Magnolia is an original genus belonging to Magnoliaceae and has a long history of cultivation in China given its beauty and fragrance. Therefore, Magnolia has been widely used in garden landscapes for its high ornamental value. Flower color is one of the main ornamental characteristics. White, pink, and purple are the main flower colors, whereas other flower colors, such as yellow and green, are uncommon (Wang et al., 2013; Zhang et al., 2011). An increase in the diversity of flower colors has become one of the main research directions of Magnolia specialists.

Received for publication 8 Aug. 2018. Accepted for publication 25 Nov. 2018.

This research was supported by the Zhejiang Provincial of China Agricultural New Varieties Breeding Major Science and Technology Projects (2016C02056-12) and the Zhejiang Forestry Promotion Project (2017B01).

${ }^{1}$ These authors contributed equally to this work. ${ }^{2}$ Corresponding author. E-mail: yameishen@zafu. edu.cn.
Flavonoids are secondary metabolites of water-soluble aromatics, with a series of two benzene rings connected by a central threecarbon chain, C6-C3-C6 (Zhao and Tao, 2015). Natural biological flavonoids are derivatives of their basic structures, and they often exist in the form of glycosides (Heiss et al., 2010). The different structural flavonoids can be divided into 10 types, including anthocyanin, flavone, flavonol, flavanone, flavanol, chalcone, isoflavone, dihydroflavonol, aurone, and proanthocyanidin (Hao et al., 2015; Tahara, 2007). Anthocyanins confer a diverse range of flower colors, including orange, red to purple, and violet (Tanaka et al., 2008), thus determining the flower color of $\approx 88 \%$ of the family angiosperms (Grotewold, 2006). Approximately 600 anthocyanins have been isolated and identified from nature, and their main derivatives are from six anthocyanin structures (Kong et al., 2003). In addition, colorless or light yellow flavonols and flavones, as copigments of anthocyanins, generally play a supplementary role in flower color (Asen et al., 1971; Ono et al., 2010). The diversity of flavonoids, which is determined by different metabolic pathways, is the fundamental cause of many flower colors (Tanaka and Brugliera, 2013). The targeted color modification of plants is conducted through the combination of the main pigment, the synthesis of the metabolic pathway, and genetic engineering (Chen et al., 2017; Tai et al., 2014).

In previous research, only one type of anthocyanin, cyanidin 3-O-glucoside chloride, was identified in Magnolia sprengeri, which was found to be the main pigment in this purpleflowered phenotype (Shi et al., 2015, 2014). Six types of flavonoids have been identified from the ethanol extracts of white-flowered Magnolia grandiflora petals, including apigenin $8-C$ glucoside, luteolin $8-\mathrm{C}$-glucoside, quercetin 3- $O$ rutinoside, quercetin 3-O-galactoside, quercetin 3-O-glucoside, and kaempferol 3-O-rutinoside, whereas the flavonoids in the stamens have been identified as isorhamnetin 3-O-glucoside and isorhamnetin (Sokkar et al., 2014). However, there is no systematic study on the flavonoid composition and color of Magnolia petals.

The purpose of the present study was to integrate and compare the flavonoid composition formation patterns of Magnolia in purple, red, pink, yellow, and white flowers. Ten Magnolia specimens, including six typical species and four corresponding cultivars, were investigated to analyze their flavonoids by high-performance liquid chromatography coupled with diode array detection (HPLC-DAD) as well as HPLC with electrospray ionization and mass spectrometry (HPLC-ESI-MS ${ }^{2}$ ). The results of the present study suggest a relationship between the components and the different flower colors in Magnolia. These results might provide some information for color breedings in Magnoliaceae.

\section{Materials and Methods}

Plant materials. Ten plant materials, including six species and four cultivars (Fig. 1), were planted in Zhejiang Agriculture and Forestry University, Lin'an, China (located at long. $118^{\circ} 51^{\prime}$ to $119^{\circ} 52^{\prime} \mathrm{E}$, lat. $29^{\circ} 56^{\prime}$ to $30^{\circ} 23^{\prime} \mathrm{N}$ ). All plant materials were chosen from one tree, and three flowers were picked from the trees at random. One petal from each flower was selected. The outermost petals of the flowers at the full-bloom stage were used as materials in this study. The flowers were cut off with their branches and placed in water in the laboratory. The color parameters of some flowers were measured immediately, whereas some petals were quickly frozen in liquid nitrogen and stored at $-80^{\circ} \mathrm{C}$ for future analysis.

Flower color parameter measurements. The use of the Royal Horticultural Society Color Chart (RHSCC) gave the ability to classify colors. The RHSCC values were assigned by comparing the base of the fresh petals (the darkest part) under the same external lighting conditions. At the same time, the color parameter lightness $\left(L^{*}\right)$ and chromatic component $\left(a^{*}\right.$ and $b^{*}$ ) values of the CIE (French Commission internationale de l'éclairage) $L^{*} a * b^{*}$ color coordinates were 

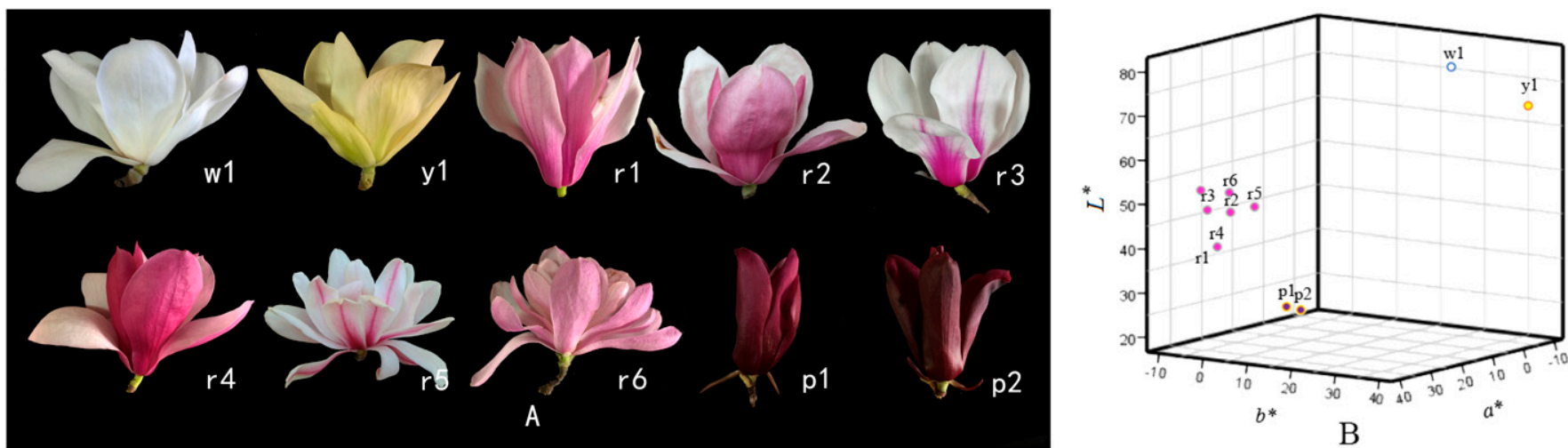

Fig. 1. (A) Ten plant materials (w1 Magnolia denudata; y1 M. denudata 'Fei Huang'; r1 Magnolia $\times$ soulangeana 'Fu Rong'; r2 M. ×soulangeana 'Dan Xin'; $\mathrm{r} 3$ Magnolia cylindrical; r4 M. Xsoulangeana; $\mathrm{r} 5$ Magnolia sinostellata; r6 Magnolia stellata 'Chrysanthemumiflora'; p1 Magnolia liliiflora; $\mathrm{p} 2$ M. liliiflora 'Hong Yuanbao'). (B) Color distribution in CIE $L^{*} a^{*} b^{*}$ coordinate systems of trivariate.

Table 1. Color parameters of 10 plant materials.

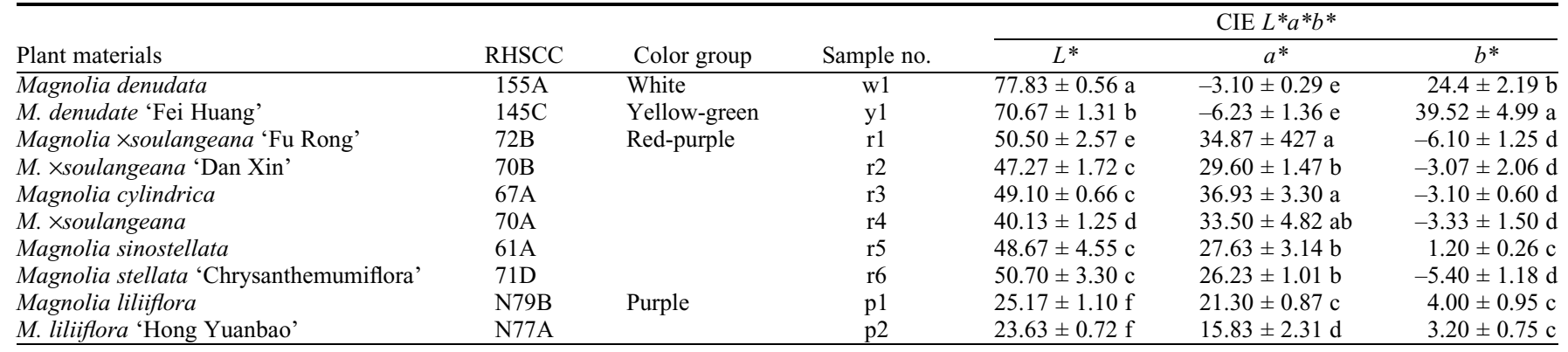

$\mathrm{CIE}=$ French Commission internationale de l'éclairage; RHSCC $=$ Royal Horticultural Society Color Chart; $L^{*}=$ lightness; $a^{*}, b^{*}=$ chromatic components.

measured using the color reader CR-10 (Konica Minolta Optics, Inc., Sakai, Japan). $L^{*}$ represents the lightness of the color, with the value ranging from low to high and the color from lighter to darker. The $a^{*}$ value represents the red (positive) and green (negative) values. The $b^{*}$ value represents yellow (positive) and blue (negative) values (Biolley and Jay, 1993).

Extraction of the flavonoids. Fresh petals $(0.5 \mathrm{~g})$ were ground into a powder with liquid nitrogen, and then $1.5 \mathrm{~mL}$ of $2 \%$ formic acid (Shanghai, China) and 70\% methyl alcohol (Ohio, USA) were added. The mixture was placed into a $2-\mathrm{mL}$ centrifuge tube and centrifuged at $10,000 \mathrm{~g}$ for $10 \mathrm{~min}$ at $4{ }^{\circ} \mathrm{C}$ to obtain the supernatant. The flavonoid extract solution was obtained after passing the mixture through a $0.22-\mu \mathrm{m}$ syringe filter into the sample bottles (Shi et al., 2015).

Qualitative and quantitative analysis of the flavonoids. A quantitative analysis of the flavonoids was conducted using a Shimadzu HPLC System (Kyoto, Japan) consisting of an LC-20AT pump, an SPD-M20A DAD detector, a CTO-10AS VP column oven, and a SIL-20A auto injector. A $10-\mu \mathrm{L}$ aliquot of each sample was eluted through a $\mathrm{C} 18$ column of Inertsil ODS-SP $(4.6 \mathrm{~mm} \times$ $250 \mathrm{~mm}, 5 \mu \mathrm{m})$ at a column temperature of $30{ }^{\circ} \mathrm{C}$ and a flow rate of $0.8 \mathrm{~mL} \cdot \mathrm{min}^{-1}$. The mobile phase consisted of solvent A, $0.1 \%$ formic acid water, and solvent $\mathrm{B}, 0.1 \%$ formic acid acetonitrile (Ohio, USA). The gradient elution programs used were as follows: 0 to 5 $\min , 0 \%$ to $5 \% \mathrm{~B} ; 5$ to $15 \mathrm{~min}, 5 \%$ to $15 \% \mathrm{~B} ; 15$ to $25 \mathrm{~min}, 15 \%$ to $23 \% \mathrm{~B} ; 25$ to $35 \mathrm{~min}, 23 \%$ to

Table 2. Identification of anthocyanins in Magnolia with high-performance liquid chromatography with electrospray ionization and mass spectrometry.

\begin{tabular}{lccccl}
\hline Peak & $\mathrm{t}_{\mathrm{R}}(\min )$ & $\lambda_{\max }(\mathrm{nm})$ & {$[\mathrm{M}]^{+}(\mathrm{m} / \mathrm{z})$} & Fragment $(\mathrm{m} / \mathrm{z})$ & \multicolumn{1}{c}{ Tentative compound } \\
\hline P1 & 16.43 & 515 & 757 & $595,449,287$ & Cyaindin-glucosyl-rhamnosyl-glucoside \\
P2 & 19.28 & 516 & 771 & $609,463,301$ & Peonidin-glucosyl-rhamnosyl-glucoside \\
P3 & 23.23 & 517 & 595 & 449,287 & Cyanidin-glucosyl-rhamnoside \\
P4 & 26.44 & 518 & 609 & 463,301 & Peonidin-glucosyl-rhamnoside \\
\hline
\end{tabular}

$26 \% \mathrm{~B} ; 35$ to $52 \mathrm{~min}, 26 \%$ to $40 \% \mathrm{~B}$; 52 to 57 $\mathrm{min}, 40 \%$ to $5 \% \mathrm{~B}$; and 57 to $65 \mathrm{~min}, 5 \%$ to $0 \%$ $\mathrm{B}$. The ultraviolet diode array detection spectra were scanned from 190 to $800 \mathrm{~nm}$. Chromatograms were obtained at $350 \mathrm{~nm}$ and $520 \mathrm{~nm}$ simultaneously.

An HPLC-ESI-MS² analysis for the qualitative measurement of the flavonoids was performed using a Thermo Fisher ion trap mass spectrometer (Waltham, MA) equipped with an electrospray ionization (ESI) interface. The HPLC separation conditions were the same as those mentioned previously. The MS conditions were as follows: the anthocyanins were applied in positive ion (PI) modes, and the other flavonoids were applied in negative ion (NI) modes. The ESI source was as follows: $\mathrm{N}_{2}$ was the drying gas and nebulizing gas, with a capillary temp of $300{ }^{\circ} \mathrm{C}$; flow rate, $8.0 \mathrm{~L} \cdot \mathrm{min}^{-1}$; ion spray voltage, $3500 \mathrm{~V}$; the capillary offset and exit voltage were $4 \mathrm{~V}$ and $5 \mathrm{~V}$, respectively, for PI and $-33 \mathrm{~V}$ and $-35 \mathrm{~V}$, respectively, for NI; and the range of $m / z$ was 100 to 1000 for the full-scan MS analysis.

The flavonoid composition of presumption depended on the MS databased on the method of Zhang et al. (2013). The determination of the content of the flavonoid components was carried out with an external standard method. The reference substances were cyanidin 3-O rutinoside chloride, quercetin 3-O-glucoside, and kaempferol 3-O-rutinoside, which were purchased from Sigma-Aldrich (St. Louis, MO). By drawing a standard curve for the semiquantitative measurement, cyanidin chloride was used as the anthocyanin standard, whereas quercetin 3-O-rutinoside was used as the flavonol standard.

Statistical analysis. Data are expressed as the means \pm the SD of the three replicates. Microsoft Office Excel 2016 and IBM SPSS Statistics 19.0 (SPSS, Chicago, IL) were used for data processing and analysis. Origin 9 was used to draw diagrams. A Pearson correlation analysis was used to analyze the relationship among the color parameters $\left(L^{*}, a^{*}\right.$, and $b^{*}$ values), anthocyanins, and flavonols [Cy, Pn, $\mathrm{Is}, \mathrm{Qu}, \mathrm{Km}$, total anthocyanin content (TA), and total flavonol content (TF)] across 10 plant materials.

\section{Results}

Flower phenotype determination of the Magnolia. According to the RHSCC and the $L^{*}, a^{*}$, and $b^{*}$ values, the 10 plant materials 
Table 3. Identification of flavonols in Magnolia with high-performance liquid chromatography with electrospray ionization and mass spectrometry.

\begin{tabular}{|c|c|c|c|c|c|}
\hline Peak & $\mathrm{t}_{\mathrm{R}}(\min )$ & $\lambda_{\max }(\mathrm{nm})$ & {$[\mathrm{M}]^{-}(\mathrm{m} / \mathrm{z})$} & Fragment $(\mathrm{m} / \mathrm{z})$ & Tentative compound \\
\hline$\overline{\mathrm{F} 1}$ & 11.65 & 244,323 & 353 & 191,179 & No tentative identification \\
\hline $\mathrm{F} 2$ & 17.80 & 243,324 & 353 & 191,179 & No tentative identification \\
\hline F3 & 23.04 & 244,329 & 947 & $785,623,461,315$ & Isorhamnetin-rhamnosyl-glucosyl-glucosyl-glucoside \\
\hline F4 & 24.01 & 246,329 & 785 & $623,461,315$ & Isorhamnetin-rhamnosyl-glucosyl-glucoside \\
\hline F5 & 24.96 & 246,329 & 931 & $769,623,461,315$ & Isorhamnetin-rhamnosyl-glucosyl-rhamnosyl-glucoside \\
\hline F6 & 26.56 & 246,329 & 769 & $623,461,315$ & Isorhamnetin-rhamnosyl-glucosyl-rhamnoside \\
\hline F7 & 27.00 & 246,329 & 805 & $769,623,461,315$ & Isorhamnetin-rhamnosyl-glucosyl-rhamnoside \\
\hline F8 & 28.67 & 246,329 & 623 & 461,315 & Isorhamnetin-rhamnosy-glucoside \\
\hline F9 & 29.42 & 254,357 & 609 & 463,301 & Quercetin 3-O-rutinoside \\
\hline F10 & 30.30 & 253,352 & 463 & 301 & Quercetin 3-O-glucoside \\
\hline F11 & 32.04 & 246,330 & 753 & 617,285 & Kaempferol derivative \\
\hline F12 & 33.04 & 245,329 & 593 & 447,285 & Kaempferol 3-O-rutinoside \\
\hline
\end{tabular}

were divided into four color groups: the white group (w1), the yellow-green group (y1), the red-purple group (r1-r6), and the purple group (p1 and $\mathrm{p} 2$ ). In three-dimensional space distribution, the $b^{*}$ and $L^{*}$ values of the white group and the yellow-green group were significantly higher than those of the red-purple group and the purple group, and the $L^{*}$ value of the red-purple group was significantly higher than that of the purple group. The $a^{*}$ value of the red-purple group was significantly higher than those of the three other groups (Fig. 1, Table 1).

Identification of the anthocyanins in Magnolia. The anthocyanins had a characteristic absorption peak at $520 \mathrm{~nm}$, with four components isolated from the flavonoid extraction solutions. Components $\mathrm{P} 1$ and $\mathrm{P} 3$ had fragment ions $287\left[\mathrm{Y}_{0}\right]^{+}$, corresponding to cyanidin, and components $\mathrm{P} 2$ and $\mathrm{P} 4$ had fragment ions $301\left[\mathrm{Y}_{0}\right]^{+}$, corresponding to peonidin, thus showing that $\mathrm{P} 1$ and $\mathrm{P} 3$ are cyanidin derivatives, whereas $\mathrm{P} 2$ and $\mathrm{P} 4$ are peonidin derivatives. MS showed that $\mathrm{P} 1$ had molecular ion $\mathrm{m} / \mathrm{z} 757[\mathrm{M}]^{+}$and fragment ions $\mathrm{m} / \mathrm{z}$ 595[M-162] $]^{+}$, 449[M-162-146] ${ }^{+}$ and $287[\mathrm{M}-162-146-162]^{+}$, thus indicating that $\mathrm{P} 1$ is a cyanidan-glucosyl-rhamnosylglucoside (Cy-GRG). P2 had molecular ion $\mathrm{m} / \mathrm{z} 771[\mathrm{M}]^{+}$and fragment ions $\mathrm{m} / \mathrm{z} 609[\mathrm{M}-$ $162]^{+}, 463[\mathrm{M}-162-146]^{+}$, and 301[M-162$146-162]^{+}$, thus indicating that $\mathrm{P} 2$ is a peonidin-glucosyl-rhamnosyl-glucoside (PnGRG) (Fumi et al., 2012; Pedreschi and Cisneros-Zevallos, 2007; Zhang et al., 2012). P3 had molecular ion $\mathrm{m} / \mathrm{z} 595[\mathrm{M}]^{+}$ and fragment ions $\mathrm{m} / \mathrm{z} 449[\mathrm{M}-146]^{+}$and 287 $[\mathrm{M}-146-162]^{+}$, thus indicating that P3 was determined to be a cyanidin-glucosyl-rhamnoside (Cy-GR). P4 had molecular ion m/z 609[M] ${ }^{+}$ and fragment ions $\mathrm{m} / \mathrm{z} 463[\mathrm{M}-146]^{+}$and 301 $[\mathrm{M}-146-162]^{+}$, thus indicating that $\mathrm{P} 4$ was a peonidin-glucosyl-rhamnoside (Pn-GR) (Liu et al., 2013) (Table 2, Supplemental Fig. 1).

Identification of the flavonols in Magnolia. Under a wavelength of $350 \mathrm{~nm}, 12 \mathrm{com}$ ponents were isolated from the flavonoid extraction solution, of which 10 were identified, including six isorhamnetin derivatives, two quercetin derivatives, and two kaempferol derivatives. Using MS, components $\mathrm{F} 1$ and $\mathrm{F} 2$ had quasi molecular ion $\mathrm{m} / \mathrm{z}$ $353[\mathrm{M}-\mathrm{H}]^{-}$and fragment ions $\mathrm{m} / \mathrm{z} 191$ and 179. There was no characteristic ion of flavonoids, so the accurate composition of $\mathrm{F} 1$ and F2 could not be inferred. However, through

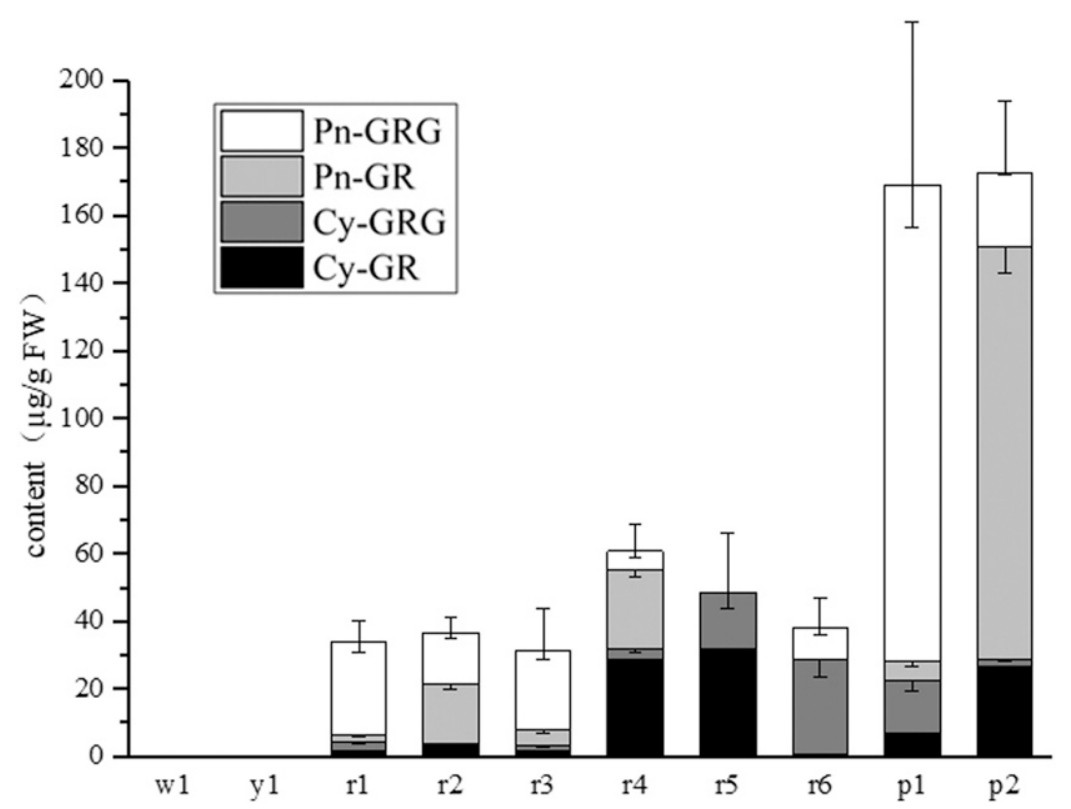

Fig. 2. Anthocyanin contents in Magnolia. $\mathrm{Cy}-\mathrm{GR}=$ cyanidin-glucosyl-rhamnoside; $\mathrm{Cy}-\mathrm{GRG}=$ cyanidinglucosyl-rhamnosyl-glucoside; Pn-GR = peonidin-glucosyl-rhamnoside; $\mathrm{Pn}-\mathrm{GRG}=$ peonidin-glucosylrhamnosyl-glucoside.

the similarity of the quasi molecular ions, a structural isomer was speculated. F3, F4, F5, F6, F7, and F8 had fragment ions $315\left[\mathrm{Y}_{0}\right]^{-}$in the NI mode corresponding to the mass spectrum characteristics of isorhamnetin, thus indicating that they were isorhamnetin derivatives. The fragment ions indicated that the type of glycosides in isorhamnetin included rhamnoside and glucoside ( $\mathrm{Li}$ et al., 2013). F9 and F10 had fragment ions 301 $\left[\mathrm{Y}_{0}\right]^{-}$and were thus speculated to be quercetin derivatives. F9 had quasi molecular ion $\mathrm{m} / \mathrm{z} 609[\mathrm{M}-\mathrm{H}]^{-}$and fragment ions $\mathrm{m} / \mathrm{z}$ 463[M-146] $]^{-}$and 301[M-146-162] $]^{-}$, and the retention time of $\mathrm{F} 9$ was the same as that of the reference substance, quercetin 3-O-rutinoside. Thus, F9 was speculated to be quercetin 3-O-rutinoside (Qu3Ru) (Wang et al., 2016). F10 had quasi molecular ion $\mathrm{m} / \mathrm{z}$ $463[\mathrm{M}-\mathrm{H}]^{-}$and fragment ion $\mathrm{m} / \mathrm{z} 301[\mathrm{M}-$ $162]^{-}$, and the retention time of F10 was the same as that of the reference substance, quercetin 3-O-glucoside. Therefore, F10 was speculated to be quercetin 3-O-glucoside (Qu3G) (Sokkar et al., 2014). F11 and F12 had fragment ion $285\left[\mathrm{Y}_{0}\right]^{-}$in the reversephase HPLC and the same glycosides. The elution time of kaempferol was later than that of quercetin (Li et al., 2009), and according to the elution sequence, it was speculated that F11 and F12 were kaempferol derivatives. F11 had quasi molecular ion $\mathrm{m} / \mathrm{z} 753[\mathrm{M}-\mathrm{H}]^{-}$and fragment ions $\mathrm{m} / \mathrm{z} 617$ and 285 ; thus, we could not infer its accurate composition. F12 had quasi molecular ion $\mathrm{m} / \mathrm{z} 593[\mathrm{M}-\mathrm{H}]^{-}$and fragment ions $\mathrm{m} / \mathrm{z}$ 447[M-146] $]^{-}$and 285[M-146$162]^{-}$, and the retention time of F10 was the same as that of the reference substance, kaempferol 3-O-rutinoside. It was therefore speculated that F12 was kaempferol 3-Orutinoside (Km3G) (Sokkar et al., 2014) (Table 3, Supplemental Fig. 2).

Composition analysis of the anthocyanins and flavonols in Magnolia. All 10 materials contained isorhamnetin (Is), quercetin (Qu), and kaempferol $(\mathrm{Km})$, but the specific flavonol composition in each plant material was different. There were no anthocyanins in the white and yellow-green groups. The red-purple group and the purple group both had anthocyanins. The main anthocyanin in Magnolia ×soulangeana 'Fu Rong', $M$. × soulangeana 'Dan Xin', and Magnolia cylindrical was peonidin (Pn). Magnolia sinostellata had only cyanidin (Cy), mainly Cy-GR. For Magnolia stellata 'Chrysanthemumiflora', the main 
pigment was Cy, mainly Cy-GRG. Last, in $M$. $\times$ soulangeana, $\mathrm{Cy}$ and $\mathrm{Pn}$ were almost the same. In the purple group, the main pigment in Magnolia liliiflora was Pn-GRG, whereas it was Pn-GR in M. liliiflora 'Hong Yuanbao'.

Compared with the other groups, the TA in the purple group was significantly higher, and the TF was significantly lower. The TF [4836.99 $\mu \mathrm{g} \cdot \mathrm{g}^{-1}$ fresh weight $\left.(\mathrm{FW})\right], \mathrm{Qu}$ $\left(2115.48 \mu \mathrm{g} \cdot \mathrm{g}^{-1} \mathrm{FW}\right)$, and $\mathrm{Km}\left(330.50 \mu \mathrm{g} \cdot \mathrm{g}^{-1}\right.$ FW) in Magnolia denudata 'Fei Huang' were higher than those in the other groups. The highest Is was in Magnolia cylindrical (2813.67 $\left.\mu \mathrm{g} \cdot \mathrm{g}^{-1} \mathrm{FW}\right)$, whereas the lowest TF was in M. liliiflora 'Hong Yuanbao' (1283.28 $\left.\mu \mathrm{g} \cdot \mathrm{g}^{-1} \mathrm{FW}\right)$, followed by $M$. liliiflora (1273.96 $\left.\mu \mathrm{g} \cdot \mathrm{g}^{-1} \mathrm{FW}\right)$ (Fig. 2, Table 4).

Relationship between the color parameters and the flavonoids. The correlation analysis results showed that TA and Pn had a highly significant negative correlation with $L^{*}$. The accumulation of TA could reduce the lightness of the petal, especially Pn. $L^{*}$ showed a significantly positive correlation with $\mathrm{TF}$ and had a greatly positive correlation with Qu. The accumulation of flavonols could increase the lightness of the petal, especially $\mathrm{Qu}$. However, the anthocyanins or flavonols showed no significant correlation with $a^{*}$ and $b^{*}$ (Table 5).

The auxiliary effect index CI value (Copigment index $=\mathrm{TF} / \mathrm{TA}$ ) was greater than 5 in 10 plant materials, suggesting that flavonols played an evident auxiliary pigment function.

\section{Discussion}

Relationship between the flavonoids and the formation of new cultivars of Magnolia. $M$. denudata was the only species with pure white flowers. Yellow flowers in Magnolia are rare outside of the North American cucumber magnolia (Magnolia acuminata). M. denudata 'Fei Huang' is a cultivar of $M$. denudata selected because of its unusual yellow flowers.

In some flowers, yellow-to-orange flower colors are attributed to carotenoids, chlorophylls, or other flavonoids, such as flavones, flavonols, and chalcones (Lewis et al., 1998; Mizuno et al., 2015). This study showed that there was no anthocyanin in $M$. denudata or $M$. denudata 'Fei Huang', and that the components and contents of the flavonols were similar in $M$. denudata and M. denudata 'Fei Huang'. The results suggest that flavonols were probably not responsible for the yellow flower color in Magnolia. Carotenoids or chlorophylls might exist in the petals of M. denudata 'Fei Huang', resulting in the yellow flower color. Nonetheless, further research is needed.

Red-purple is the most common color in Magnolia, and thus in this experiment, we chose six typical species with red-purple flowers of Magnolia. Among them, $M$. $\times$ soulangeana is a hybrid species obtained by crossbreeding $M$. denudata and M. liliiflora. The composition of anthocyanin in $M$. Xsoulangeana flowers is the same as that in M. liliiflora, as both have four anthocyanin components, whereas the TA content in $M$. $\times$ soulangeana is nearly two-fifths that of $M$. liliiflora. The formation of this new variety is due to the changes in TA.

$M$. Xsoulangeana ' $\mathrm{Fu}$ Rong' and $M$. $\times$ soulangeana 'Dan Xin' are cultivars of $M$. $\times$ soulangeana. The flower color is from a moderate purplish-red to a strong reddishpurple. In contrast to $M$. × soulangeana, the TA in $M$. × soulangeana 'Fu Rong' and $M$. $\times$ soulangeana 'Dan Xin' was reduced, especially $\mathrm{Cy}$, whereas TF had no obvious changes. The difference between $M$. × soulangeana ' $\mathrm{Fu}$ Rong' and $M$. Xsoulangeana 'Dan Xin' was the scale of Pn-GR and Pn-GRG. Thus, it can be concluded that the formation of these two new varieties is caused by the change in the anthocyanin composition and content.

M. sinostellata and M. stellata 'Chrysanthemumiflora' were once considered to be one species (Fan et al., 2018). According to this experiment, the composition of anthocyanins is very different between $M$. sinostellata and M. stellata 'Chrysanthemumiflora'. In addition, they are not the same species at the pigment level. Furthermore, M. sinostellata is an endangered Magnolia shrub species. We hope that the endangering mechanism of $M$. sinostellata can be studied in future research based on our findings (Bradshaw and Schemske, 2003; Nakatsuka et al., 2008).

M. liliiflora is a typical species of Magnolia with purple flowers, whereas M. liliiflora 'Hong Yuanbao' is a cultivar of $M$. liliiflora, with a flower color from dark purplish-red to grayish-purple. The main pigment in $M$. liliiflora is Pn-GRG, whereas it is Pn-GR in M. liliiflora 'Hong Yuanbao'. The contents of TA and TF were slightly different. The glycosylation of the anthocyanins resulted in reddening (Honda and Saito, 2002 ), which can explain why the $a^{*}$ value of $\mathrm{p} 1$ is higher than that of $\mathrm{p} 2$. The formation of this new variety is caused by different glycosides in the same anthocyanins.

Genetic engineering breeding methods of Magnolia based on the flavonoids. Traditional breeding can improve flower color based on existing colors, such as withingenus crossbreeding. However, a new flower color can be achieved by genetic engineering breeding, as opposed to traditional breeding.

The anthocyanin biosynthesis pathway belongs to the flavonoid biosynthesis pathway, and includes pelargonidin, cyanidin, and delphinidin biosynthesis branches (Martin et al., 1991). In this study, only the cyanidin biosynthesis branch was demonstrated in Magnolia (Boase et al., 2010; Jin et al., 2016; Wu et al., 2016). Thus, there are several ways to improve Magnolia flower colors depending on the type of genetic engineering breeding.

First, by introducing the corresponding structural genes, one can add a biosynthesis branch other than the cyanidin biosynthesis branch to increase the pigment types in the Magnolia petals to adjust the flower color, such as the inexistence of blue Magnolia flowers; by synthesizing delphinidin for the blue hues by incorporating $\mathrm{F}^{\prime}$ 5 $^{\prime} \mathrm{H}$ (flavonoid 3', 5'-hydroxylase), one could obtain blue flowers (Jin et al., 2016; Wu et al., 2016). Second, it is possible to change the contents of

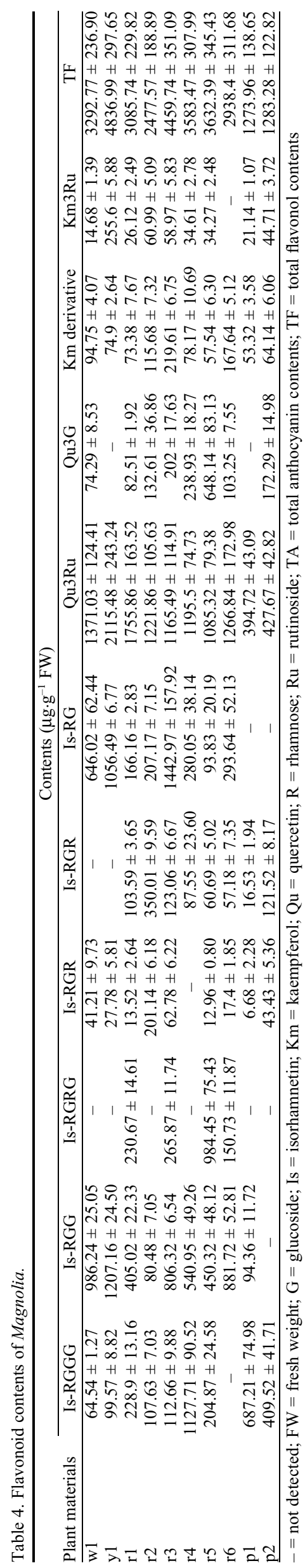


Table 5. Correlation analysis among color parameters, anthocyanins, and flavonols.

\begin{tabular}{lrrrrrrr}
\hline CIE $L^{*} a^{*} b^{*}$ coordinate & \multicolumn{7}{c}{ Pearson correlation } \\
\cline { 3 - 9 } RHSCC & \multicolumn{1}{c}{ Cy } & Pn & \multicolumn{1}{c}{ Is } & Qu & \multicolumn{1}{c}{ Km } & \multicolumn{1}{c}{ TA } & TF \\
\hline$L^{*}$ & -0.539 & $-0.832^{\mathrm{z}}$ & 0.563 & $0.765^{\mathrm{z}}$ & 0.449 & $-0.903^{\mathrm{z}}$ & $0.718^{\mathrm{y}}$ \\
$a^{*}$ & 0.322 & 0.075 & -0.064 & -0.123 & -0.252 & 0.157 & -0.113 \\
$b^{*}$ & -0.411 & -0.215 & 0.316 & 0.321 & 0.453 & -0.308 & 0.370 \\
\hline
\end{tabular}

${ }^{\mathrm{z}}$ Correlation is significant at the 0.01 level.

${ }^{\mathrm{y}}$ Correlation is significant at the 0.05 level.

$\mathrm{CIE}=$ French Commission internationale de l'éclairage; $\mathrm{Cy}=$ cyanidin; Is = isorhamnetin; $\mathrm{Km}=$ kaempferol; $\mathrm{Pn}=$ peonidin; $\mathrm{Qu}=$ quercetin; RHSCC $=$ Royal Horticultural Society Color Chart; $\mathrm{TA}=$ total anthocyanin contents; $\mathrm{TF}=$ total flavonol contents

the existing pigment components to improve flower color. For example, OMT ( $O$-methyltransferase) could catalyze Cy into Pn (Akita et al., 2011; Du et al., 2015). We speculate that the regulation of OMT could adjust the proportion of $\mathrm{Cy}$ and $\mathrm{Pn}$, with the final result being a change in Magnolia flower color.

\section{Conclusions}

The types of flavonoids in Magnolia petals are relatively few, with only two types of anthocyanin aglycones and three flavonol aglycones. There are no other flavonoids, such as flavone, and only three glycosides, including rhamnoside, glucoside, and rutinoside, exist in Magnolia petals. Cyanidin and peonidin make Magnolia petals appear red-purple and purple, respectively, whereas flavonols evidently serve as auxiliary pigments, particularly quercetin.

The formation of the cultivars of Magnolia used in this study was caused only by changes in the existing flavonoid composition rather than in the appearance of new flavonoids. The results will be valuable for flower color breeding of Magnolia.

\section{Literature Cited}

Akita, Y., S. Kitamura, Y. Hase, I. Narumi, H. Ishizaka, E. Kondo, N. Kameari, M. Nakayama, N. Tanikawa, and Y. Morita. 2011. Isolation and characterization of the fragrant cyclamen $O$ methyltransferase involved in flower coloration. Planta 234:1127-1136.

Asen, S., R.N. Stewart, and K.H. Norris. 1971. Copigmentation effect of quercetin glycosides on absorption characteristics of cyanidin glycosides and color of Red Wing azalea. Phytochemistry 10:171-175.

Biolley, J.P. and M. Jay. 1993. Anthocyanins in modern roses: Chemical and colorimetric features in relation to the color range. J. Expt. Bot. 44:1725-1734.

Boase, M.R., D.H. Lewis, K.M. Davies, G.B. Marshall, D. Patel, K.E. Schwinn, and S.C. Deroles. 2010. Isolation and antisense suppression of flavonoid 3', 5'-hydroxylase modifies flower pigments and color in cyclamen. BMC Plant Biol. 10:107.

Bradshaw, H. and D. Schemske. 2003. Allele substitution at a flower color locus produces a pollinator shift in monkeyflowers. Nature 426:176-178.

Chen, K., H. Liu, Q. Lou, and Y. Liu. 2017. Ectopic expression of the grape hyacinth (Muscariar meniacum) r2r3-myb transcription factor gene, $M a A N 2$, induces anthocyanin accumulation in tobacco. Front. Plant Sci. 8:965.

Du, H., J. Wu, K.X. Ji, Q.Y. Zeng, M.W. Bhuiya, S. Su, Q.Y. Shu, H.X. Ren, Z.A. Liu, and L.S.
Wang. 2015. Methylation mediated by an anthocyanin, $O$-methyltransferase, is involved in purple flower coloration in Paeonia. J. Expt. Bot. 66:6563-6577.

Fan, L.J., M.Q. Chen, B. Dong, N.H. Wang, Q. Yu, X.L. Wang, L.J. Xuan, Y.L. Wang, S.Z. Zhang, and Y.M. Shen. 2018. Transcriptomic analysis of flower bud differentiation in Magnolia sinostellata. Genes (Basel) 9:212.

Fumi, T., S. Norio, T. Kenjiro, S. Koichi, and H. Toshio. 2012. Flower colors and their anthocyanins in Matthiola incana cultivars (Brassicaceae). J. Jpn. Soc. Hort. Sci. 1:91-100.

Grotewold, E. 2006. The genetics and biochemistry of floral pigments. Annu. Rev. Plant Biol. 57:761-780

Hao, J., H. Zhu, Z. Zhang, S.L. Yang, and H. Li 2015. Identification of anthocyanins in black rice (Oryza sativa L.) by UPLC/Q-TOF-MS and their invitro and invivo antioxidant activities. J. Cereal Sci. 64:92-99.

Heiss, C., C.L. Keen, and M. Kelm. 2010. Flavanols and cardiovascular disease prevention. Eur. Heart J. 31:2583-2592.

Honda, T. and N. Saito. 2002. Recent progress in the chemistry of poly acylated anthocyanins as flower color pigments. Heterocycles 56:633692.

Jin, X., H. He, W. Lu, S. Yi, and S. Dai. 2016. Transcriptomics and metabolite analysis reveals the molecular mechanism of anthocyanin biosynthesis branch pathway in different Senecio cruentus cultivars. Front. Plant Sci. 7:1307.

Kong, J.M., L.S. Chia, N.K. Goh, T.F. Chia, and R. Brouillard. 2003. Analysis and biological activities of anthocyanins. Phytochemistry 64: 923-933.

Lewis, D.H., S.J. Bloor, and K.E. Schwinn. 1998. Flavonoid and carotenoid pigments in flower tissue of Sandersonia aurantiaca (Hook.). Scientia Hort. 3:179-192.

Li, C.H., H. Du, L. Wang, Q. Shu, Y. Zheng, Y. Xu, J. Zhang, J. Zhang, R. Yang, and Y. Ge. 2009. Flavonoid composition and antioxidant activity of tree peony (Paeonia section moutan) yellow flowers. J. Agr. Food Chem. 57:84968503.

Li, C.H., S. Huang, and M. Huang. 2013. Labellum colors of Oncidium alliance and their flavonoid composition. Chin. J. Trop Crop. 34:1133-1138.

Liu, N., G. Sun, Y. Xu, Z. Luo, Q. Lin, X. Li, J. Zhang, and L. Wang. 2013. Anthocyanins of the genus of Hosta and their impacts on tepal colors. Scientia Hort. 150:172-180.

Martin, C., A. Prescott, S. Mackay, J. Bartlett, and E. Vrijlandt. 1991. Control of anthocyanin biosynthesis in flowers of Antirrhinum majus. Plant J. 1:37-49.

Mizuno, T., A. Uehara, and D. Mizuta. 2015. Contribution of anthocyanin-flavone copigmentation to grayed violet flower color of Dutch iris cultivar 'Tiger's Eye' under the presence of carotenoids. Scientia Hort. 186:201-206.

Nakatsuka, T., K. Sato, H. Takahashi, S. Yamamura, and M. Nishihara. 2008. Cloning and characterization of the UDP-glucose: Anthocyanin 5-Oglucosyltransferase gene from blue-flowered gentian. J. Expt. Bot. 6:1241-1252.

Ono, E., M. Ruike, T. Iwashita, K. Nomoto, and Y. Fukui. 2010. Co-pigmentation and flavonoid glycosyltransferases in blue Veronica persica flowers. Phytochemistry 7:726-735.

Pedreschi, R. and L. Cisneros-Zevallos. 2007. Phenolic profiles of Andean purple corn (Zea mays L.). Food Chem. 99:956-963.

Shi, S.G., S.J. Li, Y.X. Kang, and J.J. Liu. 2015. Molecular characterization and expression analyses of an anthocyanin synthase gene from Magnolia sprengeri Pamp. Appl. Biochem. Biotechnol. 175:477-488.

Shi, S.G., M. Yang, M. Zhang, P. Wang, Y.X. Kang, and J.J. Liu. 2014. Genome-wide transcriptome analysis of genes involved in flavonoid biosynthesis between red and white strains of Magnolia sprengeri pamp. BMC Genomics 15:706.

Sokkar, N.M., M.A. Rabeh, G. Ghazal, and A.M. Slem. 2014. Determination of flavonoids in stamen, gynoecium, and petals of Magnolia grandiflora $\mathrm{L}$. and their associated antioxidant and hepatoprotection activities. Quim. Nova 37:667-671.

Tahara, S. 2007. A journey of twenty-five years through the ecological biochemistry of flavonoids. Biosci. Biotechnol. Biochem. 71:387-404.

Tai, D., J. Tian, J. Zhang, T. Song, and Y. Yao. 2014. A Malus crabapple chalcone synthase gene, $M c C H S$, regulates red petal color and flavonoid biosynthesis. PLoS One 9:e110570.

Tanaka, Y. and F. Brugliera. 2013. Flower color and cytochromes P450. Phytochem. Rev. 368:20120432.

Tanaka, Y., N. Sasaki, and A. Ohmiya. 2008. Biosynthesis of plant pigments: anthocyanins, betalains and carotenoids. Plant J. 54:733-749.

Wang, Y.L., E. Ejder, J.F. Yang, Y.X. Kang, W. Ye, and S.Z. Zhang. 2013. New varieties of Magnolia biondii and Magnolia cylindrica (Magnoliaceae) in China. Blumea 1:33-38.

Wang, Y.G., J.X. Fu, C. Zhang, and H. Zhao. 2016. HPLC-DAD-ESI-MS analysis of flavonoids from leaves of different cultivars of Sweet osmanthus. Molecules 21:1224.

Wu, Q., J. Wu, S.S. Li, H.J. Zhang, C.Y. Feng, D.D. Yin, R.Y. Wu, and L.S. Wang. 2016. Transcriptome sequencing and metabolite analysis for revealing the blue flower formation in waterlily. BMC Genomics 17:897.

Zhang, J., C.H. Li, L.S. Wang, and F. Chen. 2013. Golden rules of separation and characterization of plant anthocyanins by high pressure liquid chromatography-tandem mass spectrometry. Food Safe Qual. Detec. Technol. 1:760-768.

Zhang, J., L.S. Wang, J.M. Gao, Y.J. Xu, L.F. Li, and C.H. Li. 2012. Rapid separation and identification of anthocyanins from flowers of Viola yedoensis and $V$. prionantha by high-performance liquid chromatography-photodiode array detectionelectrospray ionisation mass spectrometry. Phytochem. Anal. 23:16-22.

Zhang, S.Z., Y.L. Wang, Z.C. He, and E. Ejder. 2011. Genome differentiation in Magonoliaceae as revealed from meiotic pairing in interspecific and intergeneric hybrids. J. Syst. Evol. 49:518-527.

Zhao, D.Q. and J. Tao. 2015. Recent advances on the development and regulation of flower color in ornamental plants. Front. Recent Dev. Plant Sci. 6:261. 

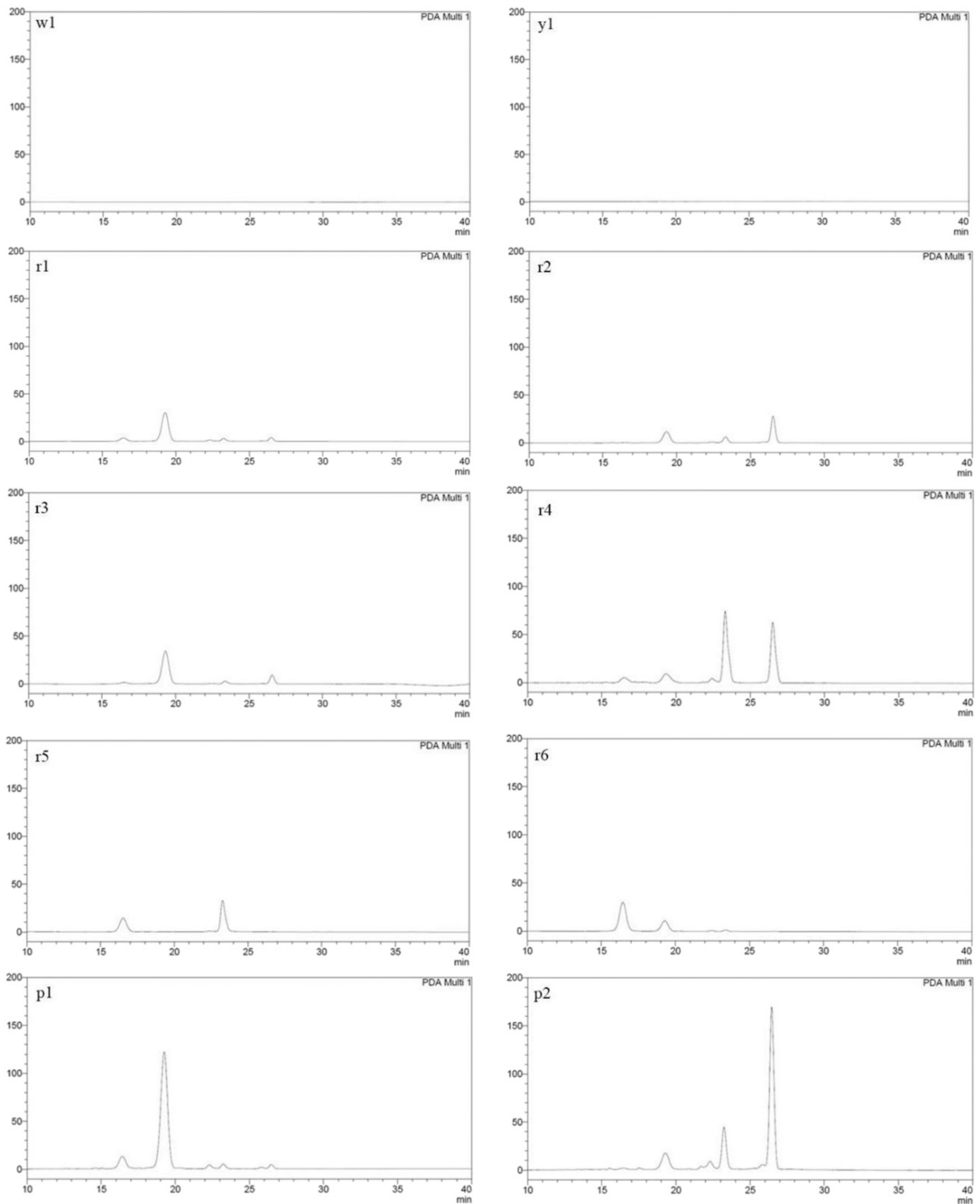

Supplemental Fig. 1. HPLC chromatogram of anthocyanins in Magnolia. 

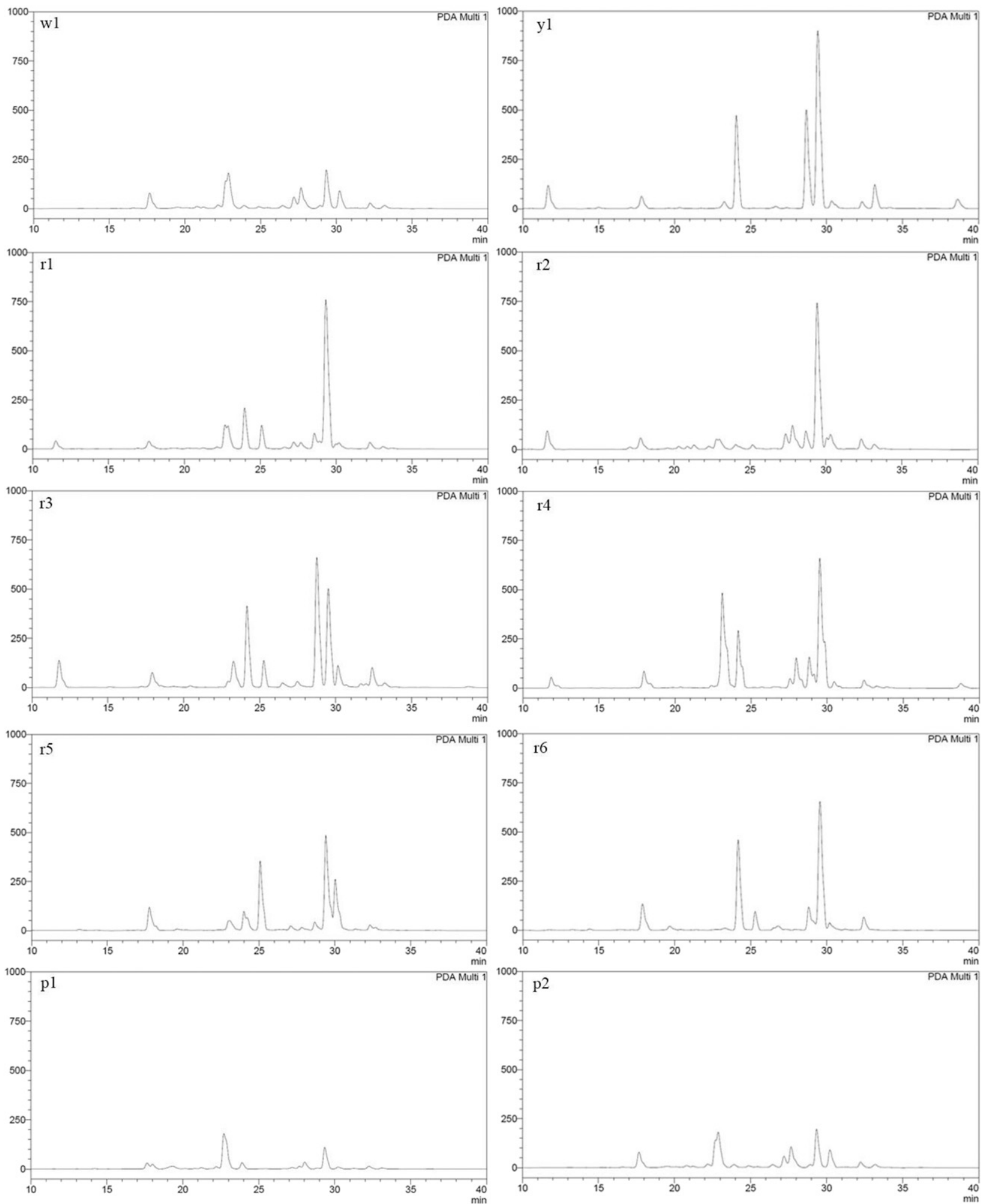

Supplemental Fig 2. HPLC chromatogram of flavonoids in Magnolia. 